\title{
Medicare Spending on Drugs and Biologics Not Recommended for Coverage by International Health Technology Assessment Agencies
}

\author{
Alexander C. Egilman, BA ${ }^{7,2}$, Joshua D. Wallach, $M S, P h D^{1,2,3}$, \\ Sanket S. Dhruva, MD, MHS , Gregg S. Gonsalves, PhD ${ }^{1,5}$, and Joseph S. Ross, \\ $M D, M H S^{1,2,6,7,8}$
}

\begin{abstract}
'Collaboration for Research Integrity and Transparency, Yale Law School, New Haven, CT, USA; ${ }^{2}$ Center for Outcomes Research and Evaluation (CORE), Yale New Haven Hospital, New Haven, CT, USA; ${ }^{3}$ Department of Environmental Health Sciences, Yale School of Public Health, New Haven, CT, USA; ${ }^{4}$ Department of Medicine, University of California, San Francisco School of Medicine, San Francisco, CA, USA; ${ }^{5}$ epartment of Epidemiology of Microbial Diseases, Yale School of Public Health, New Haven, CT, USA; '́ Section of General Medicine, Department of Internal Medicine, Yale School of Medicine, New Haven, CT, USA; 'Department of Health Policy and Management, Yale School of Public Health, New Haven, CT, USA; ${ }^{8}$ National Clinician Scholars Program, Department of Internal Medicine, Yale School of Medicine, New Haven, CT, USA.
\end{abstract}

$\mathrm{J}$ Gen Intern Med 34(11):2319-21

DOI: $10.1007 / \mathrm{s} 11606-019-05149-6$

(c) Society of General Internal Medicine 2019

\section{INTRODUCTION}

The United States (US) spends more on prescription medications than any other country. ${ }^{1}$ Many countries have created a central health technology assessment (HTA) agency to analyze available evidence on clinical safety and efficacy, as well as cost-effectiveness, of approved medical products and then recommend whether they should be covered by their respective national health program, including Australia (Pharmaceutical Benefits Advisory Committee [PBAC]), Canada (Canadian Agency for Drugs and Technologies in Health [CADTH]), and England (National Institute for Health and Care Excellence [NICE]). In contrast, the US has no single national health program and HTA is fragmented across several uncoordinated public and private initiatives. ${ }^{2}$ The Centers for Medicare and Medicaid Services (CMS), which administers Medicare, the US' largest publicly funded health insurance program, makes both local and national coverage determinations but is statutorily prohibited from considering cost-effectiveness. ${ }^{3}$ To understand the potential implications of Medicare not having an organization that systematically conducts HTAs and cost-effectiveness analyses when making coverage determinations of drugs and biologics, we estimated recent Medicare spending on drugs and biologics not recommended for coverage by at least one of 3 prominent national HTA bodies in nations with comparable health profiles to the US, PBAC, CADTH, and NICE, and subsequently not covered by each country's respective national health programs.

Prior Presentations None.

Published online July 16, 2019

\section{METHODS}

We collected coverage recommendations for all drugs and biologics published through August 2018 by PBAC, CADTH, and NICE, which began making HTA appraisals publicly available in 2005, 2004, and 2001, respectively. We identified therapeutics not recommended for coverage across all appraisals made by each HTA agency, including those for different indications, formulations, and dosages. We then determined national coverage in each HTA agency's respective country by searching the Pharmaceutical Benefits Scheme of Australia, the Ontario Drug Benefit Formulary to represent Canada as its most populous province, and the National Health Services England drug list (v.13). We excluded therapeutics not approved by the US Food and Drug Administration (FDA), FDA approved but discontinued before 2011, or FDA approved after 2016.

Medicare provides outpatient prescription drug coverage through Part D, which covered 40.8 million beneficiaries in 2016, and covers medications administered by physicians under Part B. ${ }^{4}$ We used Medicare Part B and Part D drug utilization and spending data to estimate Medicare (from 2011 to 2016) and beneficiary out-of-pocket (from 2011 to 2015) spending on the drugs and biologics not recommended nor covered in Australia, Canada, or England. ${ }^{5}$ All calculations were performed using Microsoft Excel (v.16.11).

\section{RESULTS}

We identified 53, 67, and 14 drugs and biologics that were FDA approved prior to 2016, received only negative coverage recommendations by PBAC, CADTH, and NICE, respectively, and were not publicly covered in Australia, Canada, and England, respectively. Between 2011 and 2016, we identified Medicare spending on 44 (83.0\%), 


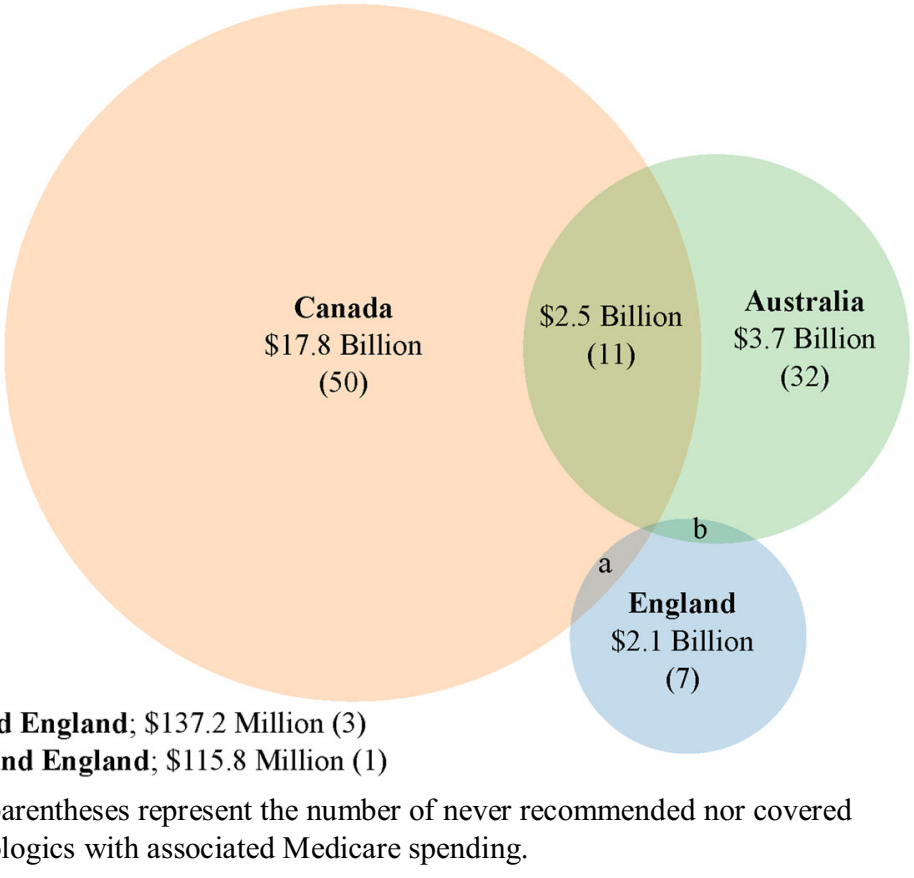

Figure 1 Medicare spending on drugs and biologics not recommended by the Pharmaceutical Benefits Advisory Committee, the Canadian Agency for Drugs and Technologies in Health, and the National Institute for Health and Care Excellence, and not covered in Australia, Canada, and England, respectively, 2011-2016.

$64(95.5 \%)$, and $11(78.6 \%)$ of these not recommended nor covered therapeutics, respectively. Medicare spending totaled $\$ 3.7 \mathrm{~B}, \$ 17.8 \mathrm{~B}$, and $\$ 2.1 \mathrm{~B}$ on drugs and biologics not recommended nor covered in only Australia, Canada, and England, respectively (Fig. 1), $\$ 2.8 \mathrm{~B}$ on those not recommended nor covered by any two countries, and $\$ 0$ by all three countries. Between 2011 and 2015, Medicare beneficiaries filled $43.4 \mathrm{M}$ prescriptions for the not

a. Canada and England; \$137.2 Million (3)

b. Australia and England; \$115.8 Million (1)

Numbers in parentheses represent the number of never recommended nor covered drugs and biologics with associated Medicare spending.

ara

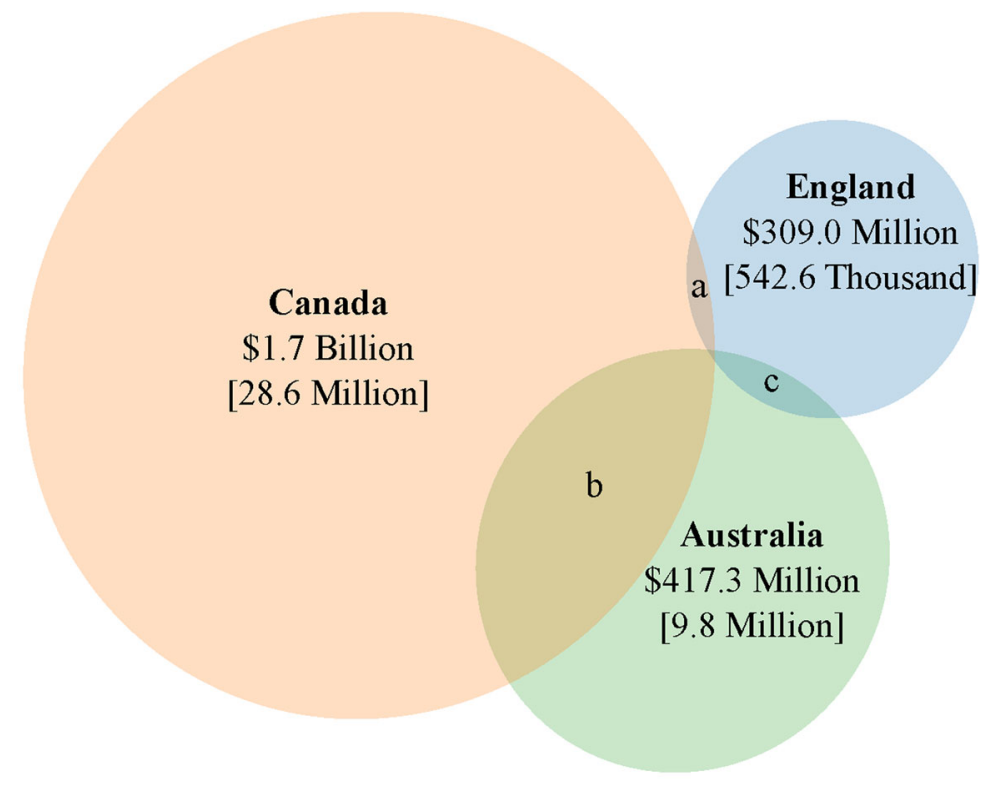

a. Canada and England; \$6.5 Million [32.8 Thousand]

b. Australia and Canada; $\$ 273.2$ Million [4.3 Million]

c. Australia and England; \$28.6 Million [90.8 Thousand]

Numbers in brackets represent the number of prescriptions filled.

Figure 2 Medicare beneficiary spending on drugs and biologics not recommended by the Pharmaceutical Benefits Advisory Committee, the Canadian Agency for Drugs and Technologies in Health, and the National Institute for Health and Care Excellence, and not covered in Australia, Canada, and England, respectively, 2011-2015. 
recommended nor covered drugs and biologics, spending \$2.8B out-of-pocket (Fig. 2).

\section{DISCUSSION}

Between 2011 and 2016, Medicare and its beneficiaries spent $\$ 29.2$ billion on 104 unique drugs and biologics that were found to lack sufficient evidence of efficacy and/or value to support coverage by the national HTA agencies of Australia, Canada, or England. Our analysis may underestimate spending since Medicare spending was limited to a 6-year period (a 5 -year period for beneficiary spending), which represents only some of the years these drugs were available to beneficiaries, and does not account for spending by Medicare Advantage plans. However, we could not account for copay coupons nor manufacturer rebates and discounts, which are substantial for some brand-name medications. Furthermore, requested prices, medications reviewed, and agency decision-making processes vary among HTA agencies and recommendations are adapted to each country's social and population needs; thus, decisions may not translate to US Medicare. Additional efforts are needed to ensure Medicare beneficiaries are prescribed drugs and biologics of the highest value.

Corresponding Author: Joseph S. Ross, MD, MHS; Section of General Medicine, Department of Internal MedicineYale School of Medicine, P.O. Box 208093, New Haven, CT 06520, USA (e-mail: joseph.ross@yale.edu).

Contributors All authors had full access to all the data in the study and take responsibility for the integrity of the data and the accuracy of the data analysis. ACE and JSR contributed to study concept and design; ACE abstracted the data; JDW and SSD verified the data; all authors contributed to the analysis and interpretation of the data; ACE drafted the manuscript; all authors contributed to the critical revision of the manuscript; and JSR provided study supervision. The corresponding author affirms that he has listed everyone who contributed significantly to the work.

Funding This project was conducted as part of the Collaboration for Research Integrity and Transparency (CRIT) at Yale, funded by the Laura and John Arnold Foundation, which supports Mr. Egilman and Drs. Wallach, Gonsalves, and Ross. Dr. Dhruva is supported by the National Clinician Scholars Program and the Department of Veterans Affairs.

Data Availability The datasets generated during the current study are available from the corresponding author on reasonable request.

\section{Compliance with Ethical Standards:}

Conflict of Interest: All authors have completed the ICMJE uniform disclosure form at www.icmje.org/coi_disclosure.pdf and declare: In the past 36 months, Dr. Wallach has received research support through the Meta Research Innovation Center at Stanford (METRICS) from the Laura and John Arnold Foundation; Dr. Gonsalves has received research support from the National Institutes of Mental Health (R01MH105203) and the National Institute on Drug Abuse (RO1DA015612); and Dr. Ross has received research support through Yale University from Johnson and Johnson to develop methods of clinical trial data sharing, from Medtronic, Inc. and the Food and Drug Administration (FDA) to develop methods for postmarket surveillance of medical devices (U01FD004585), from the Food and Drug Administration to establish Yale-Mayo Clinic Center for Excellence in Regulatory Science and Innovation (CERSI) program (UO1FD005938), from the Blue Cross Blue Shield Association to better understand medical technology evaluation, from the Centers of Medicare and Medicaid Services (CMS) to develop and maintain performance measures that are used for public reporting (HHSM-500-2013-13018I), from the Agency for Healthcare Research and Quality (RO1HSO22882), from the National Heart, Lung and Blood Institute of the National Institutes of Health (NIH) (RO1HSO25164), and from the Laura and John Arnold Foundation to establish the Good Pharma Scorecard at Bioethics International. In addition, Dr. Ross serves as an ad hoc member of the Medicare Evidence Development \& Coverage Advisory Committee (MEDCAC). Mr. Egilman and Dr. Dhruva have no additional potential competing interests to disclose.

Disclaimer: The Laura and John Arnold Foundation played no role in the design and conduct of the study; collection, management, analysis, and interpretation of the data; preparation, review, or approval of the manuscript; and decision to submit the manuscript for publication. The authors assume full responsibility for the accuracy and completeness of the ideas presented, which do not represent the views of the Department of Veterans Affairs or any other supporting institutions.

\section{REFERENCES}

1. Sarnak DO, Squires D, Kuzmak G, Bishop S. Paying for Prescription Drugs Around the World: Why Is the U.S. an Outlier? The Commonwealth Fund. 5 October 2017. Available at: http://www.commonwealthfund.org/ publications/issue-briefs/2017/oct/prescription-drug-costs-us-outlier. Accessed May 9, 2019.

2. Luce B, Cohen RS. Health technology assessment in the United States. Int J Technol Assess Health Care. 2009 Jul;25 (Suppl 1):S33-41.

3. Neumann PJ, Rosen AB, Weinstein MC. Medicare and Cost-Effectiveness Analysis. N Engl J Med. 2005 Oct 6;353(14):1516-22.

4. Hoadley J, Cubanski J, Neuman T. Medicare Part D in 2016 and Trends over Time. Henry J. Kaiser Family Foundation. 16 September 2016. Available at: https://www.kff.org/report-section/medicare-part-d-in2016-and-trends-over-time-section-1-part-d-enrollment-and-plan-availability/. Accessed May 9, 2019.

5. Centers for Medicare and Medicaid Services. Medicare Part D and Part B Spending Data. Available at https://www.cms.gov/Research-StatisticsData-and-Systems/Statistics-Trends-and-Reports/Information-on-Prescription-Drugs/MedicarePartD.html. Accessed May 9, 2019.

Publisher's Note: Springer Nature remains neutral with regard to jurisdictional claims in published maps and institutional affiliations. 